\title{
Thymic Neuroendocrine Tumor Presenting With Cushing Syndrome, As A rare initial presentation
}

\author{
ahmad matarneh ${ }^{1}$, abdelrahman hamad ${ }^{2}$, elhadi Elouzi ${ }^{2}$, Nabil mahmood ${ }^{2}$, Mohammad $^{2}$ \\ Khair Hamad ${ }^{2}$, mousa Hussein ${ }^{2}$, and Mohamed Yassin ${ }^{2}$ \\ ${ }^{1}$ Hamad medical corporation \\ ${ }^{2}$ Hamad Medical Corporation
}

September 11, 2020

\begin{abstract}
we report a 24 years old female patient, who presented with features of crushing syndrome. upon evaluation, she was found to have ectopic Adrenocorticotropic hormone (ACTH)-dependent Cushing's syndrome due to a thymic tumor. histopathology confirmed ACTH secreting tumor. she was treated With Video-assisted thoracoscopic surgery and had a successful recovery.
\end{abstract}

Thymic Neuroendocrine Tumor Presenting With Cushing Syndrome, As A rare initial presentation

Ahmad S Matarneh ${ }^{1}$, Abdelrahman O Hamad ${ }^{1}$, Mohammad K Hamad ${ }^{1}$, Elhadi B Elouzi ${ }^{2}$, Nabil S Mahmood $^{3}$, Mousa S Hussein ${ }^{4}$,Mohamed A Yassin ${ }^{5}$

1. Department of Internal Medicine, Hamad Medical Corporation, Doha, Qatar

2. Department of Endocrinology, Hamad Medical Corporation, Doha, Qatar

3. Department of Radiology, Hamad Medical Corporation, Doha, Qatar

4. Department of Pulmonology, Hamad Medical Corporation, Doha, Qatar

5. Department of Medical Oncology, Hamad Medical Corporation, Doha, Qatar

Running title: Thymic Carcinoid Tumor Causing Cushing syndrome

*Corresponding Author Dr Ahmad Samir Salam Matarneh Department Internal Medicine Hamad Medical Corporation Alrayyan street. Doha, Qatar Tel: +97455957396 Fax: -44397857 E-mail: AMatarneh@Hamad.qa

\section{Keywords:}

Neuroendocrine Neoplasm, Thymic malignancies, Cushing's disease, Pituitary Incidentaloma, HypercortisolismKey Clinical Message:while evaluating for the cause of Cushing syndrome, biochemical confirmation should be pursued first, one of the rare secondary causes that should be kept in mind is thymic neuroendocrine tum

\section{Abstract: Background:}

Thymic neuroendocrine tumors are a very rare group of neoplasms with only a few cases described in the literature. These are considered malignant predisposing them to metastasize. They can present with local symptoms or when functional secrete hormones leading to paraneoplastic syndromes such as acromegaly and Cushing's syndrome. Cushing's syndrome is characterized by signs and symptoms due to exposure to excess glucocorticoid. Establishing the diagnosis is difficult as the signs and symptoms may not be obvious. Amongst several benign and malignant causes, ectopic secretion of ACTH by non-pituitary tumors 
accounts for about 10 to 15 percent of Cushing's syndrome. Most ectopic cases are caused by neuroendocrine tumors of the lung, pancreas, or thymus. Due to diagnostic and presentation challenges, it can often be missed. We write this report to highlight a diagnostic dilemma we encountered and the association of thymic neuroendocrine tumors with Cushing's syndrome.Case report:We report a years old Filipina lady who presented to the hospital with generalized fatigue and weakness. She was found to have Cushing's syndrome and further evaluation revealed a thymic neuroendocrine tumor that was treated with surgical excision by VATS. The patient had an uncomplicated recovery course with normalization of her blood pressure and hormonal profile.Conclusion:Thymic neuroendocrine malignancies are a rare group of neoplasms that can present with cushing syndrome, it should be kept in mind while dealing with an ectopic cushing syndrome. Mainstay of treatment is surgery, with a good overall survival and outcomes.

\section{Introduction}

Cushing's syndrome is a syndrome that manifests with signs and symptoms of hypercortisolism and usually affects multiple systems. Depending on the level and duration of exposure to high cortisol levels the presentation varies with the most commonly observed findings being obesity, round (moon) facies with acne, high blood pressure, and high blood sugar with multiple electrolytes derangements [1]. Moreover, the presentation might differ between men and women, where men can present at a younger age as compared to females who can present at a later age with symptoms of gonadal dysfunction such as amenorrhea and other menstrual abnormalities being more common [2] Diagnosis usually is divided into two phases. The first is to establish the diagnosis which requires a high clinical suspicion along with confirmatory laboratory studies, such as high cortisol levels, and in-suppressible cortisol with the administration of low dose dexamethasone $(1 \mathrm{mg})$. The second phase is to identify the etiology, which is done by administering high dose dexamethasone ( $8 \mathrm{mg})$; failure to suppress cortisol indicates an ectopic source of ACTH secretion [3] The treatment choice depends on the underlying etiology. One of the secondary causes of Cushing's syndrome is neuroendocrine tumors of the thymus. These tumors may secrete ACTH leading to the rapid development of hypercortisolism and ultimately Cushing's syndrome [4]. Thymic malignancies are a rare group of diseases composing around $1 \%$ of all malignancies. Of these malignancies, thymic neuroendocrine tumors comprise $2-5 \%$ and are considered the least common [4] They inherently occur more in males with a median age of diagnosis around the 5th decade of life [5]. These are usually aggressive and malignant with the potential to metastasize. Hence treatment should be prompt to remove the thymus gland. The response is variable depending on the prognostic factors[5] Case presentation:We report A 24 years old Filipina lady, who was recently diagnosed with hypertension and hypothyroidism and presented to our hospital with generalized fatigue and weakness that had been going on for three months before presentation. She had increased abdominal girth along with acne and occasional difficulty while getting up from chairs or walking. On examination, she was thinly built with a slightly rounded face containing numerous acnes. A slight hump on her back was noted. She also had proximal bilateral weakness mainly in the lower limbs. Her Blood pressure was found to be $163 / 67 \mathrm{mmHg}$. Labs showed her $8 \mathrm{AM}$ cortisol level to be high $(2636 \mathrm{nmol} / \mathrm{l}$, Reference range $[133-537 \mathrm{nmol} / \mathrm{L}])$ with a high ACTH $(114 \mathrm{pg} / \mathrm{ml}$, Reference range $[7.2-63.3 \mathrm{pg} / \mathrm{ml}])$. A low dose dexamethasone suppression. test failed to suppress cortisol with a reading of $(3572 \mathrm{nmol} / \mathrm{l})$, but then high dose dexamethasone suppression test showed a cortisol level of (2959 nmol/l, Reference range [133-537 nmol/L]), which meant failure to suppress Cortisol suggesting the cause to be an ectopic source. A chest, abdomen and pelvic CT scan were done which showed findings of a small thymic neuroendocrine tumorFigure 1.(axial contrast-enhanced CT Study at the level of mid-thoracic regions shows a small peripherally enhancing solid abnormality within the thymus (Circle)) )associated with bilateral adrenal hypertrophy figure 2.(Axial contrast-enhanced CT study at the level of the upper abdomen shows a smooth enlargement of both adrenal glands in keeping with hypertrophy ( arrows). This further confirmed the suspected diagnosis of Cushing's syndrome secondary to a thymic neuroendocrine tumor. She underwent surgical excision of the tumor by right Video-assisted thoracoscopic surgery (VATS) with en-block excision of the mediastinal mass and total removal of anterior mediastinal fat. Samples were sent to pathology which was consistent with a typical carcinoid tumor (ACTH expressing). The tumor expressed CK AE1/AE3 and Synaptophysin that was confined to the thymus. Following the surgery, her ACTH dropped to $6.1 \mathrm{pg} / \mathrm{m}$ and cortisol to $399 \mathrm{nmol} / \mathrm{l}$, along with improvement in her symptoms and 
blood pressure with titration down on her antihypertensives with no postoperative complications.

Discussion:We present a rare case of Cushing's syndrome encountered. The initial presentation was suggestive of an endocrinopathy, which was confirmed by biochemical tests revealing hypercortisolism secondary to an ectopic source. CT scan revealed a very small tumor confined to the thymus. After resecting the tumor, the symptoms and laboratory findings related to Cushing's syndrome improved with no postoperative complications.

There is very little evidence in the literature describing the relationship of Cushing's syndrome with a thymic Neuroendocrine tumor [6]. Thymic NET's are usually rare and account for a small percentage of malignancies, occurring more in males at a the ratio of 3:1 [7].Functional Thymic tumors tend to have a more complicated course, and rapid onset of the development of clinical symptoms when associated with an endocrinopathy. Suspicion of such tumors should be raised and sought when diagnosing an ectopic Cushing's syndrome. They are detected by imaging studies, such as CT scan of the chest, FDG PET, or Dotatate PET CT which are also useful to detect any synchronous metastasis[8] Surgical excision remains the mainstay of treatment and prognosis depends on the presence of metastasis and histopathologic parameters such as mitotic activity[8]

Conclusion: While investigating the causes of Cushing's syndrome, ectopic sources should always be considered

There is limited data available on the association between Cushing's syndrome and thymic neuroendocrine tumors currently. Thymic neuroendocrine tumors are considered malignant, and the mainstay of treatment is surgical excision. Prognosis depends on several factors most crucial being that of early diagnosis and absence of distant metastasis.

Author's contribution:Ahmad S matarneh: manuscript write up, Data collection and clinical care. Adbelrahman O hamad: clinical care, study planning and design Mohammad Khair: clinical care Elhadi ouzi: clinical care Mousa S Hussein: Clinical care. Nabil sherif mahmood: clinical care Mohammad yassin: Mentor

Conflict of interest:None declared.Ethical approval: approved by Hamad medical corporation, MRC number MRC-04-20-451

Acknowledgments: We thank the internal medicine department at Hamad Medical Corporation for giving us the chance and support to conduct this work.Funding information:Qatar national library

\section{References:}

1 Sharma ST, Nieman LK, Feelders RA. Cushing's syndrome: epidemiology and developments in disease management. Clinical epidemiology. 2015;7:281.

2 Buliman A, Tataranu LG, Paun DL, Mirica A, Dumitrache C. Cushing's disease: a multidisciplinary overview of the clinical features, diagnosis, and treatment. Journal of medicine and life. 2016 Jan;9(1):12.

3 Pappachan JM, Hariman C, Edavalath M, Waldron J, Hanna FW. Cushing's syndrome: a practical approach to diagnosis and differential diagnoses. Journal of clinical pathology. 2017 Apr 1;70(4):350-9.

4.Oronsky B, Ma PC, Morgensztern D, Carter CA. Nothing but NET: a review of neuroendocrine tumors and carcinomas. Neoplasia. 2017 Dec 1;19(12):991-1002.

5 Filosso PL, Ruffini E, Solidoro P, Roffinella M, Lausi PO, Lyberis P, Oliaro A, Guerrera F. Neuroendocrine tumors of the thymus. Journal of thoracic disease. 2017 Nov;9(Suppl 15):S1484.

6 Rosai J, Higa E. Mediastinal endocrine neoplasm, of probable thymic origin, related to carcinoid tumor. Clinicopathologic study of 8 cases. Cancer. 1972 Apr;29(4):1061-74.

7 Lawrence L, Zhang P, Choi H, Ahmad U, Arrossi V, Purysko A, Makin V. A unique case of ectopic Cushing's syndrome from a thymic neuroendocrine carcinoma. Endocrinology, diabetes \& metabolism case reports. 2019 Feb 22;2019(1). 
8 Chaer R, Massad MG, Evans A, Snow NJ, Geha AS. Primary neuroendocrine tumors of the thymus. The Annals of thoracic surgery. 2002 Nov 1;74(5):1733-40.

Figures legends: figure 1: axial contrast-enhanced CT Study at the level of mid-thoracic regions shows a small peripherally enhancing solid abnormality within the thymus (Circle figure 2: Axial contrast-enhanced CT study at the level of the upper abdomen shows a smooth enlargement of both adrenal glands in keeping with hypertrophy ( arrows)

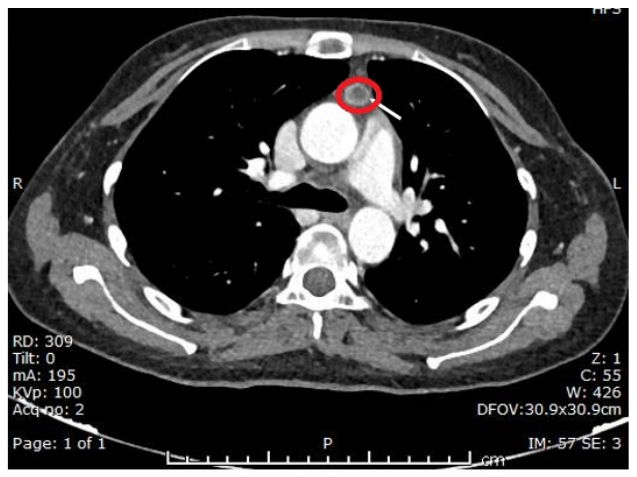

Figure 1. Axial contrast enhanced CT study at the level of the mid thoracic region shows a small peripherally enhancing solid abnormality within the thymus ( white arrow).

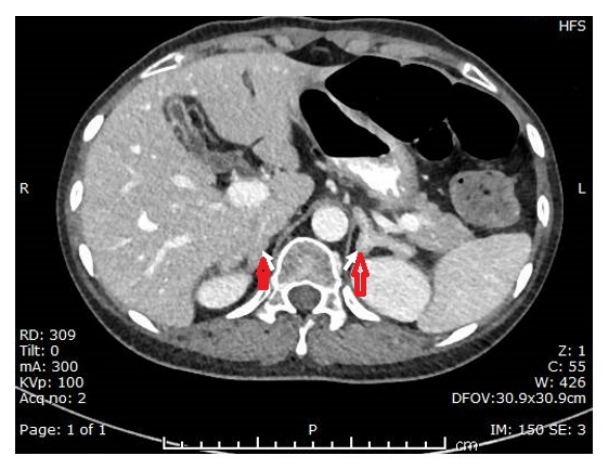

Figure 2. Axial contrast enhanced CT study at the level of the upper abdomen shows smooth enlargement of both adrenal glands in keeping with hypertrophy ( white arrows). 